Trauma Berufskrankh 2008 10 [Suppl 3]:322-326

DOI 10.1007/s10039-008-1408-7

Online publiziert: 15 . Mai 2008

(c) Springer Medizin Verlag 2008

\author{
A. Jester ${ }^{1} \cdot$ U. Hoppe ${ }^{2}$ \\ ${ }^{1}$ Klinik für Hand, Plastische und Rekonstruktive Chirurgie - \\ Schwerbrandverletztenzentrum, BG-Unfallklinik, Ludwigshafen \\ ${ }^{2}$ Abteilung für Anästhesiologie, Intenisvmedizin und Schmerztherapie, \\ BG-Unfallklinik, Ludwigshafen
}

\title{
Präklinische Erstversorgung von Brandverletzten
}

Nur 1\% aller Einsätze im Rettungsdienst betreffen Brandverletzungen. Aus diesem Grund besteht nicht selten eine Unsicherheit der Ärzte und Sanitäter bei der Versorgung dieser Patienten. Die Qualität der Erstversorgung bestimmt jedoch in einem hohen Ausmaß die weitere Morbidität und Mortalität der Betroffenen. Im Folgenden werden daher notwendige Maßnahmen der Erstversorgung verbrannter Patienten erläutert und zusammengefasst. Zum besseren Verständnis werden zuvor Verbrennungsgrade und -ausdehnung sowie die Pathophysiologie der Verbrennungserkrankung kurz erläutert.

\section{Verbrennungsgrade und -ausmaß}

Grundsätzlich werden oberflächliche Verbrennungen (Grad I, IIa) mit Verletzung der oberen Hautschichten von tiefen Verbrennungen (Grad IIb, III) unterschieden. Die erstgradige Verbrennung ist lediglich eine Hautrötung. Die Verbrennung Grad IIa ist gekennzeichnet durch einen feuchten Wundgrund, Rötung, Schwellung und Blasenbildung (Blasen präklinisch nicht eröffnen) sowie starke Schmerzen bei starker Wundsekretion. Bei einer Verbrennung Grad IIb können noch Blasen vorhanden sein, die drittgradige Verbrennung jedoch zeigt einen weißen, schmerzlosen Wundgrund. In der Regel handelt es sich um Mischbilder von oberflächlichen und tiefen Verbrennungen, wodurch die Ersteinschätzung der Verbrennungstiefe erschwert wird.

Ein wichtiger Parameter hinsichtlich der Sofortmaßnahmen ist die Ausdehnung der Verbrennung. Sie wird nach der so genannten Neunerregel nach Wallace eingeschätzt.

Tipp. Bei fleckförmigen Verbrennungen entspricht die Handfläche des Verletzten etwa 1\% seiner Körperoberfläche. Ansonsten umfassen beim Erwachsenen jeweils ein Arm 9\%, ein Bein 18\% und der Rumpf vorne und hinten jeweils $18 \%$ der Körperoberfläche. Beim Kind hat der Kopf eine größere Ausdehnung.

Die präklinische Abschätzgenauigkeit unterscheidet sich häufig um 10\% oder mehr von der präzisen Berechnung der VKOF (verbrannte Körperoberfläche) in der Klinik. Dies liegt daran, dass eindeutige Kriterien wie Blasen in der präklinischen Phase häufig noch fehlen. Im Zweifelsfall sollte die VKOF eher großzügig eingeschätzt werden.

\section{Pathophysiologie}

Eine länger dauernde Hitzeeinwirkung oder die kurzfristige Einwirkung hoher Temperaturen verursachen eine Schädigung des Hautorgans, seiner Anhangsgebilde und evtl. der darunter liegenden Gewebeanteile. Das Ausmaß einer Verbrennung wird durch die Flächenausdehnung und die Tiefe der jeweiligen Verbrennungswunden bestimmt.

$\mathrm{Zu}$ Beginn der Verletzung besteht eine erhöhte Gefäßdurchlässigkeit im verbrannten Gebiet mit den daraus resultierenden hohen Flüssigkeitsverlusten in den interstitiellen Raum und an der Verbrennungsoberfläche. Dies ist der Grund für das Auftreten des Verbrennungsödems bei Verbrennungen über $20 \%$ verbrannter
Körperoberfläche und für die so genannte Schockphase, die 48-72 h anhält. Hieraus lässt sich der sehr hohe Flüssigkeitsbedarf des Brandverletzten in den ersten $24 \mathrm{~h}$ ableiten. Mit der Schockphase entwickelt sich das vital bedrohliche Bild der Verbrennungskrankheit, das u. a. auch durch eine erhöhte Gefäßdurchlässigkeit im verbrannten und nichtverbrannten Gebiet gekennzeichnet ist.

\section{Präklinische Versorgung}

\section{Sofortmaßnahmen}

Insbesondere beim Retten aus geschlossenen Räumen ist unbedingt an den Selbstschutz zu denken. Es muss immer den Anordnungen der Feuerwehr Folge geleistet werden.

Die Rettungsmaßnahmen umfassen: Brennende oder schwelende Kleidung des Patienten wird sofort abgelöscht $z$. B. werden dem Patienten eine Baumwolldecke oder ähnliches übergeworfen bzw. dieser am Boden entlang gerollt und die Flammen auf diese Weise erstickt. Keinesfalls sollte synthetisches Material zum Ablöschen benutzen werden. Nach dem Löschen sollte die Kleidung des Patienten entfernt werden, da bei Verbrühungen der Hitzestau durch nicht entfernte verbrannte Kleidung die thermische Verletzung verstärken kann. Feuchte Textilien haben eine hohe Wärmespeicherkapazität - besonders gefährdet durch diese Verbrühungen sind Kleinkinder. Ist die Kleidung mit der Wunde verklebt, können Kleiderreste umschnitten werden. 


\section{Kaltwassertherapie}

Sie ist eine Maßnahme der Selbst- und Laienhilfe. Sie ist deshalb sofort nach dem Verbrennungstrauma, aber längstens innerhalb der ersten 15 min durchzuführen. Durch die Kühlung werden v. a. die häufig starken Schmerzen des Verbrannten gelindert; die Verhinderung eines tieferen Hitzeschadens (so genanntes Nachbrennen, „afterburn“) scheint eher von untergeordneter Bedeutung zu sein.

Die Kaltwassertherapie ist eine typische Erstmaßnahme, die von Laien ausgeführt wird und mit dem Eintreffen des Rettungsdiensts (durchschnittliche Eintreffzeit desselben 12-15 $\mathrm{min}$ ) beendet werden sollte, da der Notarzt über bessere Möglichkeiten der Schmerzlinderung als kaltes Wasser verfügt. Der Rettungsdienst konzentriert sich auf den Erhalt der Körperwärme des Verletzten, der gerade einen Teil seiner wärmeisolierenden Hautschicht und - durch die durchgeführte Kaltwassertherapie - auch Körperwärme verloren hat. Ein Auskühlen des Patienten mit deutlichem Absinken der Körpertemperatur erhöht die Mortalität deutlich.

Grundsätze der Kaltwassertherapie. Sie ist immer eine Maßnahme der Selbst- und Laienhilfe. Dabei gilt:

- Sie darf die notfallmedizinische Versorgung nicht verzögern.

- Sie darf die Prähospitalzeit nicht verlängern.

- Überspülen betroffener Areale mit $15^{\circ} \mathrm{C}$ kaltem Leitungswasser für maximal $10 \mathrm{~min}$

- Kaltwasserbäder nur bei isolierten Extremitätenverbrennungen

- Grundsätzliche Zurückhaltung bei Kleinkindern (schnellere Auskühlung)

Kontraindikationen. Die Kaltwassertherapie darf nicht angewendet bzw. muss sofort beendet werden bei:

- beginnender Auskühlung (Kältezittern, „shivering“)

- Verbrennung mit Begleitverletzung ( thermomechanisches Kombinationstrauma)

- großflächiger Verbrennung von mehr als $30 \%$ VKOF

Trauma Berufskrankh 2008 · 10 [Suppl 3]:322-326 DOI 10.1007/s10039-008-1408-7

(c) Springer Medizin Verlag 2008

\section{A. Jester $\cdot$ U. Hoppe \\ Präklinische Erstversorgung von Brandverletzten}

\section{Zusammenfassung}

Eine adäquate Primärversorgung brandverletzter Patienten am Unfallort kann die Überlebensprognose bereits maßgeblich verbessern. Nach Bergen aus dem unmittelbaren Gefahrenbereich und initialer kurzfristiger Kühlung muss der Verletzte nach Entfernen aller Kleidung gewärmt werden, um eine Auskühlung zu verhindern. Anschließend werden zunächst die Vitalfunktionen des Betroffenen gesichert. Das exakte Einschätzen von Verbrennungsausdehnung und -tiefe steuert den sinnvollen Einsatz der Infusionstherapie (Baxter-Formel) und der Analgesie. Patienten, bei denen Verbrennungen im Gesicht, an den Händen, Füßen oder der Anogenitalregion vorliegen, müssen in ein Verbrennungszentrum verlegt werden. Ebenso Pati-

enten mit mehr als $15 \%$ verbrannter Körperoberfläche Grad II oder 10\% Grad III, Patienten mit mechanischen Begleitverletzungen und/ oder Inhalationstrauma, Patienten unter 8 Jahre oder älter als 60 Jahre, des Weiteren alle Patienten mit elektrischen Verletzungen. Sollte eine Verlegung in ein Spezialzentrum nicht innerhalb von 30 min möglich sein, muss in einem anderen, näher gelegenen Krankenhaus erstversorgt werden, um dann den Patienten stabilisiert in ein entsprechendes Zentrum weiterverlegen zu können.

\section{Schlüsselwörter}

Präklinische Erstversorgung · Brandverletzung · Kühlung/Wiederaufwärmung . Infusion · Überlebensprognose

\section{Preclinical treatment of burn victims}

\section{Abstract}

Adequate primary care of burned individuals at the scene of an accident can significantly improve their survival prognosis. After the patient is rescued from immediate danger, short-term cooling is helpful but should not be prolonged. Afterward, all of the patient's clothing should be removed, the patient warmed, and vital functions secured. Accurate estimation of burn extent and depth determines the appropriate use of infusion therapy (Baxter formula) and analgesia. A person with burns to the face, hands, feet, or anogenital region must be relocated to a specialized burn center. Other patients requiring transfer to a burn unit include those with 2nd-degree burns covering more than $15 \%$ body surface, 3rd-degree burns covering more than $10 \%$ body surface, patients with mechanical injuries and/or inhalation trau$\mathrm{ma}$, and patients under 8 years of age or older than 60 years, as well as victims of electrical trauma. If transport to a specialized center is not possible within $30 \mathrm{~min}$, the patient must be transported to a nearby hospital for initial stabilization before being relocated to a burn unit.

\section{Keywords}

Preclinical primary care · Burn injury Cooling/warming up $\cdot$ Infusion $\cdot$ Survival prognosis 
Merke: Nach der Hitze droht die Kälte! Deshalb muss an einen Wärmeschutz (Schutz vor weiterer Auskühlung) des Patienten gedacht werden: Die verbrannten Körperteile sollten mit Sekret aufnehmendem Verbandsmaterial, Metallinetüchern und Rettungsdecken bedeckt werden. Bei ungünstigen Witterungsverhältnissen sollten angewärmte Infusionslösungen sowie die Heizung des Rettungswagens zum Einsatz kommen!

\section{Wundversorgung}

Die Versorgung der Verbrennungswunde beschränkt sich in der präklinischen Phase auf die „sterile“ Abdeckung mit Sekret aufnehmendem, nicht verklebendem Material. Es darf keine „Reinigung“ der ohnehin initial sterilen Verbrennungswunde stattfinden, Brandblasen sollen nicht eröffnet oder abgetragen werden. Auf die Lokalbehandlung mit Salben, Antiseptika oder Ähnlichem ist bei der Verbrennungswunde zu verzichten.

\section{Infusionstherapie}

$\mathrm{Ab}$ einer Verbrennungsausdehnung bei Erwachsenen von mehr als 15\% VKOF bzw. bei Greisen und Kindern von mehr als $10 \%$ VKOF ist zur Verhinderung eines Schockgeschehens immer eine Infusionstherapie erforderlich. Verletzten mit geringeren Verbrennungsausdehnungen (<10\% VKOF) kann ausnahmsweise oral Flüssigkeit zugeführt werden, eine Infusionstherapie erscheint aber auch in diesem Fall sinnvoll.

Für das Anlegen von venösen Zugängen bei Brandverletzten gelten folgende Grundsätze:

- maximal 2 großlumige periphere Venen punktieren (Gefahr der Überinfusion)

- großlumige Kanülen verwenden (14 G oder $16 \mathrm{G}$ )

- i.v. Zugänge nicht nur durch Pflaster, sondern auch durch Mullbinden und Schienen sichern, evtl. mit Naht fixieren (zirkuläre Verbände vermeiden Gefahr der Abschnürung der Blutversorgung der Extremität)

- grundsätzlich unverbrannte Regionen für die Venenpunktion bevorzugen
- Punktion in Ausnahmefällen auch im keimarmen verbrannten Gebiet möglich (beachte: Venen im verbrannten Areal sind häufig thrombosiert)

- primär keine Indikation für ZVK (zentraler Venenkatheter) wegen der hohen Infektionsgefahr

Die Lösung zur Infusionstherapie in den ersten $24 \mathrm{~h}$ ist nach den derzeit gültigen Empfehlungen der deutschsprachigen Arbeitsgemeinschaft für Verbrennungsbehandlung (DAV) Ringer-Laktat oder jede andere Vollelektrolytlösung. Die Infusionsmenge der ersten 24 h errechnet sich näherungsweise (!) aus der geschätzten Ausdehnung der Verbrennung und dem geschätzten Körpergewicht des Patienten nach der so genannten Parkland-Formel von Baxter: Die 24-h-Menge an RingerLaktat entspricht $4 \mathrm{ml} \times \mathrm{kg} \mathrm{KG} \times \mathrm{VKOF}$ (KG: Körpergewicht).

Ein einfaches Rechenbeispiel soll den Volumenbedarf in der präklinischen Phase erläutern: ein etwa $80 \mathrm{~kg}$ schwerer Patient mit 50\% VKOF benötigt 16.000 $\mathrm{ml}$ RingerLaktat in den ersten $24 \mathrm{~h}$, davon die Hälfte in der ersten 8 h, d. h. $1000 \mathrm{ml} / \mathrm{h}$.

Merke: Faustregel für den Infusionsansatz. Ein etwa $80 \mathrm{~kg}$ schwerer Erwachsener mit 50\% VKOF erhält 1 1/h RingerLaktat.

Zur Vermeidung der häufig zu beobachtenden massiven Überwässerung von Brandverletzten in der präklinischen Phase ist die Infusionsmenge der genannten Parkland-Formel in keinem Fall zu überschreiten.

Eine Kreislaufinsuffizienz, die trotz korrekter verbrennungsspezifischer Volumensubstitution weiterbesteht, ist ein deutlicher Hinweis auf eine Begleitverletzung (so genanntes thermomechanisches Kombinationstrauma, etwa 5\% aller Schwerbrandverletzten). In diesem Fall gelten die notfallmedizinischen Prinzipien der Versorgung eines Mehrfachverletzten.

Keinesfalls darf der Verbrannte noch an der Unfallstelle katheterisiert werden: Zwar dient die stündliche Urinausscheidung als Maß für die suffiziente Volumentherapie, aber das Infektionsrisiko einer präklinischen Harnröhrenkatheterisierung ist bei den ohnehin sehr infekti- onsgefährdeten Verbrennungsopfern sehr hoch.

Zusammenfassend sind alle bekannten „Verbrennungsformeln“ für die Flüssigkeitstherapie lediglich Orientierungshilfen - die tatsächliche Flüssigkeitssubstitution muss sich immer am Bedarf des Patienten orientieren.

\section{Analgesie und Anästhesie}

Die stärksten Schmerzen werden durch großflächige Grad-II-Verbrennungen hervorgerufen, bei tiefen Grad-III-Verbrennungen bestehen aufgrund der Zerstörung nozizeptiver Strukturen häufig keine Schmerzen. Offensichtlich verbrannte Haut, die sich schmerzfrei präsentiert, gibt einen Hinweis auf die Tiefe der Verbrennung!

Eine schnell und sicher wirksame Analgesie kann durch Opioide wie Morphin oder Piritramid erfolgen, wobei bei dem spontan atmenden Patienten auf die mögliche Atemdepression geachtet werden muss. Eine sehr wirksame und sichere Analgosedierung am Unfallort kann durch Ketamin in vorsichtiger Kombination mit einem Benzodiazepin wie Midazolam erfolgen.

\section{Inhalationstrauma}

Dieser Begriff beschreibt eine akute Schädigung v. a. der Lunge. Ihr Ausmaß hängt von der Dauer der Exposition, der Art des verbrannten Materials sowie der Konzentration und der Löslichkeit der Substanzen ab (wichtig: Unfallhergang erfragen!). Nach der auslösenden Noxe lassen sich 3 Arten des Inhalationstraumas unterscheiden.

\section{Systemische Inhalationsvergiftung}

Sie ist häufig und erfolgt v. a. durch Kohlenmonoxid (CO) und Zyanid.

CO-Vergiftung. Sie ist eine der häufigsten unmittelbaren Todesursachen bei Bränden, die Substanz entsteht bei jeder unvollständigen Verbrennung organischen Materials. Die Symptomatik einer CO-Intoxikation reicht von Übelkeit und Schwindel bis hin zur Desorientiertheit und tiefen Bewusstlosigkeit. Das klassische „kirschrote“ Hautkolorit tritt beim 
Brandverletzten häufig in den Hintergrund und wird leicht übersehen. In jedem Fall muss dem CO-Vergifteten Sauerstoff über die Gesichtsmaske (Fluss 61 / min) zugeführt werden, der bewusstlose Patient ohne Schutzreflexe wird intubiert und beatmet.

Die Beatmung sollte wie bei jedem Notfallpatienten immer mit $100 \% \mathrm{O}_{2}$ und einem endexspiratorischen Überdruck (Peep) erfolgen. Durch die hohe inspiratorische Sauerstoffkonzentration wird das an den Erythrozyten gebundene CO schneller wieder durch Sauerstoff verdrängt. Die Pulsoxymetrie zur Beurteilung der arteriellen Sauerstoffsättigung ist durch die falsch-positive Anzeige bei einer CO-Vergiftung (so genannte Dyshämoglobine) nicht aussagekräftig. Bei schweren CO-Vergiftungen erscheint die sekundäre Verlegung in eine Klinik mit der Möglichkeit der hyperbaren Sauerstofftherapie (HBO) sinnvoll.

Zyanidvergiftung. Die Toxizität der Zyanidgase beruht auf der Hemmung des oxidativen Zellstoffwechsels, die Folge ist bei entsprechend hohen Zyanidkonzentrationen am Brandort eine innere Erstickung des Vergifteten.

Die Symptome einer primär überlebten Blausäurevergiftung ähneln denen einer CO-Intoxikation, die Therapie besteht ebenfalls in einer großzügigen Sauerstoffgabe. Vor der unkritischen, d. h. prophylaktischen Anwendung des Zyanidantidots und Methämoglobinbildners 4DMAP wird ausdrücklich gewarnt.

\section{Thermisches Inhalationstrauma}

Es entsteht durch direkte Hitzeeinwirkung und zeichnet sich durch eine ausgeprägte Schleimhautschwellung im Rachenbereich aus. Die Schädigung der tiefen Atemwege durch Hitze ist selten, da die eingeatmete heiße Luft bis zur Trachea weitgehend abgekühlt ist. Nur bei der Einatmung von gespanntem Wasserdampf ist aufgrund dessen hoher Wärmespeicherfähigkeit mit Hitzeschäden im unteren Atemtrakt zu rechnen.

Eine Schwellung der oberen Atemwege führt nur in absoluten Ausnahmefällen bereits am Unfallort zur potenziell tödlichen Atemwegsverlegung. Meist kündigt sich die zunehmende Obstruktion durch hitzebedingte Schleimhautschwellung mit gesteigerter Atemnot und einem inspiratorischen Stridor an. Bei diesen Patienten darf mit der frühzeitigen elektiven Intubation nicht gezögert werden.

\section{Chemisches Inhalationstrauma}

Es entsteht durch das Einatmen von toxischen Verbrennungsprodukten natürlicher und synthetischer Materialien. Es kann je nach Wasser- bzw. Fettlöslichkeit der Substanz zu tief gelegenen Störungen des Tracheobronchialsystems kommen.

Die Vergiftungssymptome treten entweder sofort (Reizgase vom Soforttyp) oder einige Stunden verzögert auf (Reizgase vom Latenztyp).

Der therapeutische Effekt einer systemischen oder inhalativen Zufuhr von Kortikosteroiden (z. B. inhalative Gabe von Auxiloson) ist nicht bewiesen und mehr als fraglich. Bei Brandverletzten mit begleitendem Inhalationsschaden der Lunge überwiegt wahrscheinlich der immunsuppressive Effekt des Kortisonpräparats gegenüber der erhofften entzündungshemmenden Wirkung. Damit wird der ohnehin infektionsgefährdete Brandverletzte wahrscheinlich zusätzlich geschädigt. Außerdem muss bei der notfallmäßigen Anwendung von Dosieraerosolen bezweifelt werden, ob die Substanzen überhaupt ihren Wirkort in der Lunge erreichen.

Bei im Vordergrund stehender Bronchospastik sollten neben der obligaten $\mathrm{O}_{2}{ }^{-}$ Gabe Bronchospasmolytika in typischer Weise zum Einsatz kommen.

Behandlungsgrundsätze bei Inhalationstrauma. Wichtige Punkte sind:

- Bereit beim klinischen Verdacht auf ein Inhalationstrauma ist eine großzügige $\mathrm{O}_{2}$-Gabe indiziert.

- Bei mechanischer Atemwegsobstruktion durch Schwellung bzw. bei Bewusstlosigkeit sollte frühzeitig eine endotracheale Intubation erfolgen.

- Die Beatmung muss immer mit 100\% $\mathrm{O}_{2}$ durchgeführt werden. Die Pulsoxymetrie liefert falsch-hohe Werte.

- Keine prophylaktische 4-DMAP-Gabe bei Verdacht auf Zyanidintoxikation

- Fraglicher Effekt der inhalativen Gabe von Kortikoiden

\section{Thermomechanische Verletzungen}

Unter einem „thermomechanischen Kombinationstrauma" versteht man einen Patienten, der neben seiner kutanen Verbrennung auch ein schweres Trauma erlitten hat. Angesichts der eindrücklichen Verbrennungen werden diese ebenfalls lebensbedrohlichen Begleitverletzungen häufig übersehen. Hinweise auf ein mechanisches Trauma ergeben sich aus der Rekonstruktion des Unfallhergangs (Verkehrsunfall, Sprung aus dem Fenster usw.).

Die Begleitverletzungen haben gegenüber der Verbrennung eindeutige Versorgungspriorität, deshalb muss das primäre Transportziel entsprechend ausgewählt werden.

Besonders gefährdend für den Verbrennungspatienten sind Verletzungen mit Einblutungen in Körperhöhlen wie das Schädel-Hirn-Trauma (SHT), die stumpfe Abdominalverletzung oder ein schweres Thoraxtrauma. Ein Spannungspneumothorax muss schnellstmöglich erkannt und bereits präklinisch durch das Einlegen einer Bülau-Dränage behoben werden. Klinisch vordringlich behandelt werden müssen Verletzungen der Wirbelsäule sowie Luxationen und Frakturen großer Röhrenknochen. Die operative Versorgung von Frakturen ist in den ersten Stunden nach dem Trauma auch durch verbranntes Gewebe hindurch möglich, dabei ist die Infektionsgefahr innerhalb der ersten $6 \mathrm{~h}$ nach der Verbrennung nur unwesentlich erhöht.

\section{Transporthinweise}

Bei ausgedehnten Verbrennungen erfolgt die Behandlung in einer Klinik mit einem angeschlossenen Zentrum für Brandverletzte (s. Bettenverzeichnis). Befindet sich eine solche Klinik nicht in unmittelbarer Nähe des Einsatzorts (etwa 30 min Transportzeit), sollte der Verbrannte in das nächste geeignete Krankenhaus mit einer chirurgischen und anästhesiologischen Versorgungsmöglichkeit gebracht werden. Von dort kann nach adäquater Erstversorgung und Stabilisierung der Vitalfunktionen ein Sekundärtransport in eine Spezialklinik für Brandverletzte stattfinden. Der Transport über größere Dis- 


\section{Infobox 1}

Zentrale Vermittlungsstelle für Schwerbrandverletzte der Berufsfeuerwehr Hamburg: 040-428513998

tanzen sollte per Sekundärhubschrauber erfolgen.

Vor der Verlegung findet eine obligate telefonische Kontaktaufnahme mit der aufnehmenden Verbrennungsklinik statt: Auskunft über die Aufnahmekapazität der Zentren und über die jeweilige Telefonnummer erteilt die zentrale Vermittlungsstelle für Schwerbrandverletzte der Berufsfeuerwehr Hamburg (• Infobox 1). Im Einzelfall empfiehlt sich mit Hilfe der Rettungsleitstelle die Zusammenarbeit mit der regional zuständigen Verbrennungsklinik, auch hier ist ein direkter telefonischer Kontakt von Vorteil.

Die Kriterien zur Verlegung Brandverletzter in eine Spezialabteilung sind:

- $>20 \%$ VKOF Grad II

- >10\% VKOF Grad III

- Alter der Patienten <10 und >6o Jahre; Patienten mit schweren Vorerkrankungen

- Beteiligung von Gesicht, Händen, Füßen, Genitalien oder sonstiger komplizierter Lokalisation (z. B. Achselhöhle)

- Begleitendes Inhalationstrauma, sonstige Begleitverletzungen

- Elektrotrauma, Hochvoltverbrennung, Verätzung

\section{Schlussfolgerung}

Die Maßnahmen, die vor Ort am Unfallort von Notarzt und Sanitäter getroffen werden, haben maßgeblichen Einfluss auf das Überleben Brandverletzter. Die Durchführung oben beschriebener Grundsätze kann die Unsicherheit bei der Primärversorgung minimieren und die Mortalität der Betroffenen verbessern.

\section{Korrespondenzadresse}

\section{Dr. A. Jester}

Klinik für Hand, Plastische und Rekonstruktive Chirurgie - Schwerbrandverletztenzentrum, BG-Unfallklinik Ludwigshafen, Ludwig-Guttmann-Straße 11, 67071 Ludwigshafen andrea.jester@urz.uni-heidelberg.de

\section{Literatur}

..... kann beim Verfasser angefordert werden

Interessenkonflikt. Keine Angaben. 